\title{
Photo-induced dynamics in bromoform molecules studied by femtosecond XUV transient absorption spectroscopy
}

\author{
Florian Lackner ${ }^{1,2,3}$, Adam S. Chatterley ${ }^{1,2}$, Benjamin W. Toulson ${ }^{1}$, Daniel M. Neumark ${ }^{1,2}$, \\ Stephen R. Leone $\mathrm{e}^{1,2,4}$, and Oliver Gessner ${ }^{{ }^{*}}$ \\ ${ }^{1}$ Chemical Sciences Division, Lawrence Berkeley National Laboratory, Berkeley, California, 94720 , \\ USA \\ ${ }^{2}$ Department of Chemistry, University of California, Berkeley, California, 94720, USA \\ 3Institute of Experimental Physics, Graz University of Technology, Petersgasse 16, 8010 Graz, Austria \\ ${ }^{4}$ Department of Physics, University of California, Berkeley, California, 94720, USA
}

\begin{abstract}
Ultrafast dissociation dynamics in bromoform molecules initiated by UV $(263 \mathrm{~nm})$ excitation and by strong-field ionization are explored using femtosecond XUV transient absorption spectroscopy.
\end{abstract}

\section{Introduction}

The ultrafast photochemistry of bromoform $\left(\mathrm{CHBr}_{3}\right)$ is currently attracting great interest [1]. Here, we employ femtosecond transient inner-shell absorption spectroscopy to probe the photoinduced dynamics at the $\mathrm{Br}_{4,5}$ edge, providing site-specific insight from the viewpoint of the $\mathrm{Br}$ atoms.

\section{Experimental}

In the UV experiments, bromoform molecules are excited by $120 \mathrm{fs}$ long, $263 \mathrm{~nm}$ "pump" pulses derived from the $3^{\text {rd }}$ harmonic of a NIR fundamental $(785 \mathrm{~nm})$. XUV "probe" pulses are generated in a high-order harmonic generation (HHG) process, producing femtosecond XUV pulses covering a photon energy range from $\approx 50 \mathrm{eV}$ to $\approx 72.5 \mathrm{eV}$ [2-4]. Pump- and probe-beams intersect in a gas cell containing bromoform at a backing pressure of $\approx 7$ mbar. The transmitted XUV light is spectrally dispersed by a grating and detected with an X-ray CCD camera. Scanning of the pump-probe time delay provides insight into the evolving dynamics on a femtosecond timescale. An overall instrument response function (IRF) of $130 \mathrm{fs}$ (FWHM) is determined by monitoring the UV-induced ponderomotive shift of $n p \longleftarrow 4 d^{-1}$ inner-shell to Rydberg transitions in Xe atoms. The UV-initiated dynamics are compared to strong-field ionization induced dynamics using the fundamental NIR laser with an IRF of $\approx 40$ fs.

\footnotetext{
* Corresponding author: ogessner@lbl.gov
} 


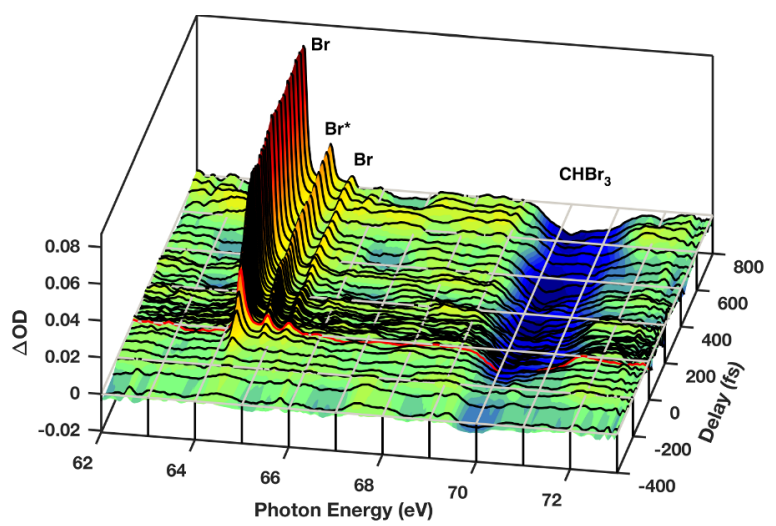

Fig. 1. Femtosecond transient inner-shell absorption spectra of bromoform $\left(\mathrm{CHBr}_{3}\right)$ molecules following excitation with $263 \mathrm{~nm}$ pulses at an intensity of $\sim 4 \times 10^{12} \mathrm{~W} / \mathrm{cm}^{2}$. Negative (blue) and positive (red) contributions correspond to the depletion of ground state parent molecules and the emergence of fragment $\mathrm{Br}$ atoms, respectively.

\section{Results and Discussion}

The static XUV absorption spectrum (not shown) is dominated by transitions from $\mathrm{Br} 3 \mathrm{~d}_{5 / 2}$ and $3 \mathrm{~d}_{3 / 2}$ core-levels into unoccupied valence orbitals, appearing as a prominent doublepeak structure around $70.5 \mathrm{eV}$. The location of these features is slightly lower in bromoform than in vinyl bromide [3] or dibromoethane [4].

Figure 1 shows transient inner-shell absorption spectra, i.e., time-dependent changes of the $\mathrm{CHBr}_{3} 3 \mathrm{~d}^{-1}$ spectrum, after excitation with a $263 \mathrm{~nm}$ pulse at a pump laser intensity of $\sim 4 \times 10^{12} \mathrm{~W} / \mathrm{cm}^{2}$. The depletion of ground state bromoform molecules around $70.5 \mathrm{eV}$ (blue regions, negative $\Delta \mathrm{OD}$ ) and the emergence of $\mathrm{Br}$ atoms (sharp peaks, positive $\Delta \mathrm{OD}$ ) are readily apparent. Based on ref. [5], the atomic $\mathrm{Br}$ peaks are assigned to core to valence transitions in ground state $\mathrm{Br}\left({ }^{2} \mathrm{D}_{3 / 2} \leftarrow{ }^{2} \mathrm{P}_{3 / 2}(64.4 \mathrm{eV}),{ }^{2} \mathrm{D}_{5 / 2} \leftarrow{ }^{2} \mathrm{P}_{3 / 2}(65.4 \mathrm{eV})\right)$ and excited state $\mathrm{Br}^{*}\left({ }^{2} \mathrm{D}_{3 / 2} \leftarrow{ }^{2} \mathrm{P}_{1 / 2}(65.0 \mathrm{eV})\right)$ products, as indicated.

The spectral signature of neutral molecular fragments is superimposed on the parent molecule depletion signal (Fig. 2a). The two spectral contributions are decomposed by determining the difference between the measured depletion signal (blue) and an inverted copy of the static parent molecule spectrum (green), scaled to the same absolute minimum. The difference spectrum (red) is dominated by two features near $\sim 71 \mathrm{eV}$ and is tentatively assigned to $\mathrm{CHBr}_{2}$ fragments based on the argument that one UV photon is sufficient to access the $\mathrm{CHBr}_{2}+\mathrm{Br}$ asymptote but two photons are required for the production of $\mathrm{CHBr}$ $+2 \mathrm{Br}$ [6]. A significant $\mathrm{CHBr}+\mathrm{Br}_{2}$ product channel contribution can be excluded due to the absence of $\mathrm{Br}_{2}$ in the spectra, which would exhibit a strong absorption feature around $68.8 \mathrm{eV}$ [7].

Fig. $2 \mathrm{~b}$ shows the temporal evolution of the parent molecule depletion (black) and the emergence of $\mathrm{Br}$ (blue) and $\mathrm{Br}^{*}$ (red) fragments. All dynamics proceed on sub-100 fs timescales, faster than the IRF. However, while a molecular fragment has been identified in the long-time delay spectrum, the present signal-to-noise ratio does not allow conclusions about transient molecular features that may be present only close to $t=0$. 
At higher UV pump intensities $\left(\sim 1 \times 10^{13} \mathrm{~W} / \mathrm{cm}^{2}\right)$, the instantaneous rise of a broad feature at $66.5 \mathrm{eV}$ is observed (not shown). This feature is only visible near zero pumpprobe delay and evolves into sharp lines for longer time delays, corresponding to the formation of $\mathrm{Br}^{+}$fragments. The UV experiments are complemented by measurements on strong-field ionized bromoform, which also leads to the formation of $\mathrm{Br}, \mathrm{Br}^{*}$ and $\mathrm{Br}^{+}$. Interestingly, however, atomic fragments form within $\sim 170$ fs after strong-field ionization, significantly slower than after UV excitation.
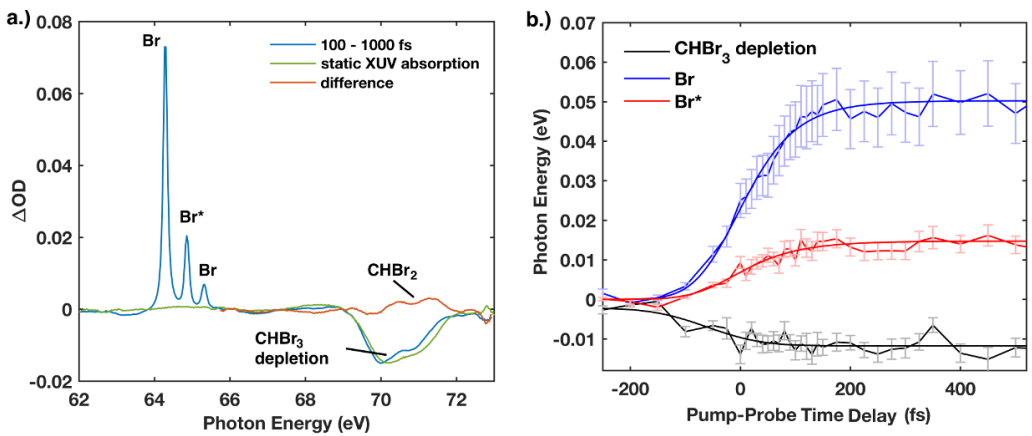

Fig. 2. UV excitation of bromoform at pump intensities of $\sim 4 \times 10^{12} \mathrm{~W} / \mathrm{cm}^{2}$ : a) Long-time delay spectrum (blue, average for $\Delta \mathrm{t}=100 \mathrm{fs} \ldots 1000 \mathrm{fs}$ ) and inverted static XUV absorption spectrum (green), scaled to match the depletion feature. The difference between the two spectra reveals the formation of a molecular fragment, which is attributed to $\mathrm{CHBr}_{2}$. b) Temporal evolution of the emergence of $\mathrm{Br}$ (blue) and $\mathrm{Br}^{*}$ (red) fragments as well as the depletion of ground state bromoform molecules (black).

\section{Acknowledgements}

This work was supported by the U.S. Department of Energy, Office of Science, Office of Basic Energy Sciences, Chemical Sciences, Geosciences and Biosciences Division, through Contract No. DE-AC02-05CH1 1231. F.L. would like to acknowledge support by the Austrian Science Fund (Grant No. J 3580-N20).

\section{References}

1. A.S. Mereshchenko, E.V. Butaeva, V.A. Borin, A. Eyzips and A.N. Tarnovsky, Nat. Chem., 7, 562 (2015).

2. F. Lackner, A.S. Chatterley, C.D. Pemmaraju, K.D. Closser, D. Prendergast, D.M. Neumark, S.R. Leone and O. Gessner, J. Chem. Phys., 145, 234313 (2016).

3. M.-F. Lin, D.M. Neumark, O. Gessner and S.R. Leone, J. Chem. Phys. 140, 064311 (2014).

4. A.S. Chatterley, F. Lackner, D.M. Neumark, S.R. Leone and O. Gessner, Phys. Chem. Chem. Phys., 18, 14644-14653 (2016).

5. A. Cummings and G. O’Sullivan, Phys. Rev. A., 54, 323 (1996).

6. S.K. Pal, A.S. Mereshchenko, E.V. Butaeva, P.Z. El-Khoury and A.N. Tarnovsky, J. Chem. Phys., 138, 124501 (2013).

7. E.R. Hosler and S.R. Leone, Phys. Rev. A 88, 023420 (2013). 